\title{
Chronically time-in or intermittently time-out? Differentiating time-out from a shortage of opportunities
}

P. L. Schwagmeyer*a, Douglas W. Mock ${ }^{\text {a }}$, Terri L. Bartlett ${ }^{\text {a }}$

${ }^{a}$ Department of Biology, University of Oklahoma, Norman, U.S.A.

27 FAX: 014053256202

28 departmental phone: 014053256200

29 home phone: 018047080874

30

email: plsch@ou.edu

31

32

Word count: 6532

33 
Males that provide parental care may lose opportunities to acquire extrapair mates or secondary social

mates. These opportunity costs can be estimated as the product of: a) the degree to which a male's participation in care makes him unready for mating (i.e., represents 'time-out' from the mating pool) and b) the availability of potential mating partners. One stage of parenting may therefore be more costly than another because it depresses a male's probability of capitalizing on any given mating opportunity, and/or because it occurs when alternative mating opportunities are more abundant. To determine if male parental care limits extrapair mating in house sparrows, Passer domesticus, we tracked changes in male extrapair mating success across stages of their nesting cycles. We then assessed whether periods of reduced extrapair mating by males coincided with a scarcity of fertilizable females, or whether certain activities placed males in time-out. We found that the number of potential extrapair mates was significantly higher on days when a male's social mate was fertile than during incubation or nestling provisioning; breeding synchrony consequently reduces the realized opportunity costs of male parenting. Nevertheless, males with nestlings to

47 feed suffered greater opportunity costs than might be expected because of their relative inefficiency at obtaining extrapair matings with potentially available females. During the incubation stage, by contrast, the males' probability of extrapair mating per fertile female averaged four times higher. Thus, nestling provisioning apparently places males in a partial time-out, but male incubation does not. Males may be more

51 fully engaged in the extrapair mating pool during incubation because they typically devote little time to this

52 form of care and, when confronted with an increase in the abundance of extrapair mating prospects, they 53 reduce the time they commit to incubation.

55 Keywords: extrapair fertilization, extrapair mating, opportunity cost, house sparrow, parental care, incubation, breeding synchrony, alternative mating opportunity, cost of reproduction 
Sexual selection models often depict individuals as alternating between periods devoted to mate

59 acquisition (engaged in, e.g., advertisement, mate searching, courtship), and periods in which individuals are occupied with mating and its aftermath (Clutton-Brock \& Parker, 1992; Kokko \& Jennions, 2008; Kokko, Klug,

61 \& Jennions, 2012; Parker \& Simmons, 1996; Simmons \& Parker, 1996 ). These latter 'time-out' intervals

62 incorporate the time required to replenish gametes, mate-guard, regenerate nuptial gifts, and/or provide 63 parental care. By definition, individuals in time-out are not prepared to mate and they are not considered participants in the mating pool (Clutton-Brock \& Parker, 1992; Simmons \& Parker, 1996); hence, if additional chances to reproduce exist during time-out, individuals experience an opportunity cost (Grafen, 1980). Time-in/time-out models are mainly heuristic, and their predictions usually hinge on comparisons of

67 relative differences in time-out durations, rather than quantitative estimates. Nevertheless, the concept of time-out invites consideration of the ways in which one reproductive event could hamper an organism's 69 capacity to engage in another, and hence incur opportunity costs. Opportunity costs may be minimal in some cases. For example, if males furnish only gametes on any reproductive event, the time necessary for

71 replenishing those may be trivial (Parker \& Simmons, 1996). Moreover, gametes or the somatic resources 72 required to produce them may be restored during periods when sexual activity does not occur (Magrath \& 73 Komdeur, 2003). Opportunity costs may also be relatively low when mate acquisition and mate-guarding, or mate acquisition and parenting, are not entirely mutually exclusive activity states (Magrath \& Komdeur, 2003;

75 Stiver \& Alonzo, 2009). Classic examples include some taxa with male-only care in which a male's mating 76 success increases once he begins undertaking parental care (Tallamy, 2000). Diverse views have been expressed about when avian males might experience especially pronounced opportunity costs in the form of lost extrapair matings. Male birds have been regarded as equally

79 physiologically competent at extrapair fertilization (EPF) regardless of the stage of their nesting cycle 80 (Stutchbury, 1998; Weatherhead, 1997); however, their ability to capitalize on mating opportunities during 81 certain stages may be diminished by constraints on their time and/or by their hormonal state, thereby placing 
82 them in a partial time-out. For instance, Westneat, Sherman, and Morton (1990) suggested that highly

83 synchronous breeding should generate lower EPF rates because fertilizable females are available only briefly

84 and because the timing of their availability can create a trade-off for males between guarding a fertile social mate and seeking EPFs. Alternatively, Ketterson and Nolan (1994) proposed that, if testosterone (T) mediates

86 a trade-off between male mating effort and parental effort, and acquisition of alternative matings is

87 facilitated by a high-T state, then opportunity costs should be especially high for males that are parenting and 88 in low-T states.

One way of detecting if particular male activity states impose greater opportunity costs than others is to examine male success in obtaining extrapair mates across successive stages of the nesting cycle. In one of the first such studies, Wetton, Burke, Parkin and Cairns (1995) found that extrapair sires in house sparrows,

92 Passer domesticus, were disproportionately likely to be older males, and that approximately half of the EPFs

93 by older males probably occurred while the male's social mate was fertile (when male T is high). On a per-

94 day basis, male EPF success was roughly twice as high when a male's social mate was fertile as it was during 95 later stages of his nesting cycle, implying that the latter stages (when males are participating in incubation 96 and nestling feeding) carry relatively steeper opportunity costs. But the source of variation in extrapair mating 97 success across stages of the breeding cycle has often been unclear, because the realized opportunity costs of each stage are the product of the number of potential mates available at that time and any stage-specific reduction in the probability that males succeed in mating on any given opportunity. For instance, males may have lower EPF success while feeding nestlings than when their mates are fertilizable simply because

101 breeding is highly synchronous and more opportunities occur during their mates' fertile periods (Canal, 102 Jovani, \& Potti, 2012; Westneat \& Gray, 1998). Thus, any trade-offs these males encounter during the two 103 stages, or any limits on their readiness-to-mate, may have little impact on the differences in their extrapair 104 mating success. Indeed, Wetton et al. (1995) cited breeding synchrony as the explanation for the high EPF 105 success of house sparrow males whose social mates were fertile. 
Here, we have repeated the Wetton et al. (1995) study by identifying extrapair sires in a North

107 American population of house sparrows, and then comparing the estimated timing of EPFs relative to the 108 stage of each extrapair sire's own nesting cycle (i.e., his 'activity state'). In a first set of analyses, we examined 109 the occurrence of successful extrapair mating in relation to male activity state, as Wetton et al. (1995) had 110 done, and we tested whether males were equally likely to obtain extrapair matings during each state. We 111 then assessed whether the observed changes across states in extrapair mating success are attributable to 112 variation in the abundance of potential extrapair mating partners, or whether there are also changes across 113 activity states in the males' incidence of successful extrapair mating per potential mate. A result indicating that the males' probability of extrapair mating per fertile female varies across stage of the nesting cycle would imply that male house sparrows are not always equally prepared to mate, i.e., that 116 they undergo periods of at least partial time-out from the extrapair mating pool. It might also suggest that 117 males expend more effort on extrapair mate acquisition while in some activity states than they do while in 118 others, or that the effort expended is especially effective in fertilization. However, such interpretations 119 assume that the females that are fertilizable at different stages of male cycles are drawn randomly, and do 120 not differ in terms of their propensity for extrapair mating from females that become fertile at other times. 121 This may not be the case. Some females may be more prone toward extrapair mating than others (e.g., 122 Forstmeier, 2007; Whittingham, Dunn, \& Stapleton, 2006), and if that variation is related to the relative 123 timing of their breeding, then females that are predisposed toward engaging in extrapair mating may 124 confront a male mating pool that is biased toward males in certain states. Consequently, in a second set of 125 analyses, we examined the composition of the pool of males present when the female participants in 126 extrapair matings were fertile. We then tested whether these females showed any bias toward mating with 127 males in certain states given the relative abundance of males in each state. If the occurrence of female extrapair matings with males in various activity states simply tracks the numbers of males in each state, then 

conducted at a second site (Schwagmeyer, Parker, Mock, \& Schwabl, 2012). Birds at the unmanipulated site

there is no need to implicate state-related changes in male effectiveness at acquiring extrapair matings as an explanation for state-related differences in male extrapair mating success.

In general, when male efforts toward acquiring extrapair mates conflict with their concurrent involvement in a separate activity, they are expected to resolve the dilemma according to the likely fitness consequences of each alternative option (e.g., Ketterson \& Nolan, 1994; Magrath \& Komdeur, 2003). In a final component of this study, we tested this hypothesis by focusing on male parental contributions during the incubation stage, which our analyses revealed to be characterized by an especially high incidence of extrapair mating. Previous research has shown that individual males of some avian species respond facultatively to changes in the abundance of fertile females, reducing the time they spend incubating on days when the pool of potential extrapair mates expands (Magrath \& Elgar, 1997); the increase in the potential gain derived from male investment in extrapair mate acquisition presumably offsets any harmful effects of prolonged male absence from his nest. We predicted that male house sparrows would show this same responsiveness to fertile female availability during incubation, given that females of this species compensate partially for reductions in male incubation contributions (Schwagmeyer, Bartlett, \& Schwabl, 2008; Schwagmeyer, Schwabl, \& Mock, 2005), and males are not particularly competent at warming the eggs, anyway (Parker, Schwagmeyer, \& Mock, 2014).

\section{METHODS}

Background

House sparrows are multi-brooded, non-migratory and non-territorial, defending only the area immediately surrounding their nesting site. We used data collected during 2000-2003 at a study site that we intentionally left unmanipulated as a complement to testosterone manipulations being concurrently are predominantly socially monogamous, but some polygyny occurs as well ( 12\% of pairs: Schwagmeyer, 
2014); around 17\% of offspring are a result of EPF (Edly-Wright, Schwagmeyer, Parker, \& Mock, 2007). During the years of this study we had a total of 38 nestboxes mounted on buildings, fences, and utility poles in an area of around $14 \mathrm{ha}$. The minimum distance between any two nestboxes was approximately $3 \mathrm{~m}$, and the maximum was around $590 \mathrm{~m}$. We banded nearly all adults that bred in nestboxes, as well as any 11-day old offspring they produced, with USGS aluminium bands and unique combinations of colour bands. A small ( $100 \mu$ l) blood sample was collected from each bird at the time it was banded. To determine clutch sizes, laying dates, hatching dates, and fledging success, we censused the contents of nestboxes at least twice per week during the breeding season unless the nest contained chicks that were nearing fledgling age ( $>11$ day old); we avoided disturbing such chicks until we could verify their absence from the nest.

\section{Parentage Exclusion and Assignments}

We followed the protocol described in Edly-Wright et al. (2007) for paternal exclusions, and we used the procedures described in Schwagmeyer et al. (2012) to assign extrapair sires. Briefly, we first used multilocus DNA fingerprinting to identify extrapair young (EPY). We then screened potential extrapair sires (all nestbox resident males, plus any males that had not used a nestbox for breeding but were captured in mistnets or ground traps) for each of the EPY at two microsatellite loci, and reduced the number of potential sires to males that matched the EPY at both loci. We then repeated the DNA fingerprinting analyses for further exclusion of potential extrapair sires. The focal extrapair sires in this study thus consisted of individuals that were the only male of those considered that matched at the two microsatellite loci and met the criteria of 0.53 or greater band-sharing with the focal chick plus fewer than two unattributable bands. Because our aim was to compare male extrapair mating success in relation to the other reproductive activities in which he was engaged, we omitted from our sample of extrapair sires two males that had sired EPY during intervals in which we lacked information about whether the male had a social mate and/or lacked information about the stage of his nesting cycle (one male did not begin breeding in a nestbox until more than 40 days after laying had commenced that year, and so may have been breeding elsewhere when he sired EPY; another 
177

male sired EPY after his social mate had ceased breeding for the season and we had no separate evidence indicating that he was still present at the study site). Our final sample of extrapair sires consisted of 20 different males (5 sires in 2000, 8 in 2001, 8 in 2003), some of which had more than one extrapair mate per season and/or sired EPY in more than one year.

Focal male dataset

We categorized the activities of our 20 focal extrapair sires during each day of the relevant breeding season in terms of four activity states (Fig. 1). We defined a female's fertile period as beginning 5 days prior to the date the first egg of her clutch was laid and lasting through the day the penultimate egg in the clutch was laid. [The actual fertile period of female house sparrows is not known; we selected this definition because of its prevalence in the passerine literature (e.g., Akçay et al., 2011; Hammers et al., 2009; Neudorf, Stutchbury, \& Piper, 1997 ).] Beginning with the first day that a female became fertile each year, and ending on the last day of the fertile period of the last female to lay eggs that season, we classified each male as: 1) having a fertile social mate; 2 ) incubating (assumed to begin on the day after the last egg was laid and to continue for the next 10 days; or 3) nestling feeding (beginning on the following day and continuing for the next 10 days.) Finally, 4) a male was considered prefertile during days when: a) breeding at the study site had commenced but the male's mate was not yet fertile (or he had not yet attracted a mate); b) male engagement in nestling feeding had ended but he had not yet entered a next 'fertile' mate stage (i.e., the interbrood interval); and c) the male was known to be alive, but his last nesting cycle for the season ended before the seasonal termination of fertile female availability. The durations of the activity states of each male were adjusted to accommodate any disruptions of the typical schedule that were created by nest failures. Some focal males also had unknown activity states during particular intervals, usually toward the end of the breeding season when we could not be certain they were still alive; we excluded these intervals from the analyses. Two focal males were polygynous during a portion of the breeding season in which they sired EPY, although both were socially monogamous at the time of the extrapair mating. To assign them to a single activity state on days 
201

when they were polygynously paired, we gave priority to the schedule of the female that had become fertile more recently. For example, if female A had finished laying and begun incubating when female B became fertile, the male was categorized as having a fertile social mate; once female A became fertile again, he was classified according to her state. (Polygynous male house sparrows do not consistently allocate parental care according to the order in which they paired with their social mates: Veiga, 1990).

Each focal male's extrapair mating success in each state during each of his nesting cycles was scored as the number of different females (that had been fertile during the interval in question) with which the male had sired extrapair offspring (range $=0-2$ ). (We used the number of different extrapair mates of a male as our measure of extrapair mating success because we thought it reasonable to assume that fertilization of one or more eggs of a particular female was independent of fertilization of another female's eggs.) When the fertile period of a male's extrapair mate overlapped two different activity states of the male, we considered the mating to have occurred during the state that included the greater number of days in which the female was fertile. In the sole case where an extrapair female had been fertile an equal number of days in two of her partner's activity states, we (arbitrarily) assigned the mating to the later-occurring state. To assess differences across states in the number of potentially available extrapair mates, we tallied the total number of different females (other than the male's social mate) at the study site that had been fertile during all or part of each activity state of each male during each of his nesting cycles. Although the maximum distance between the nesting site of a focal male in this study and his extrapair mate was $\sim 320 \mathrm{~m}$, we chose to consider all nestbox residents in estimating the size of the extrapair mating pool for two reasons. First, preliminary analyses showed little indication of any substantial variation among the temporal distributions of the fertile periods of females residing within different sub-areas of the study; thus, changes across male states in the number of fertile females on the study area were highly likely to reflect changes in fertile female abundance at a local level. Second, we did not want to assume that there was zero chance of encounter between a male and female whose nesting sites were, for example, separated by more than $320 \mathrm{~m}$ or, 
alternatively, located in different sub-areas. (Indeed, we have often observed birds flying $>250 \mathrm{~m}$ from their nesting site to forage in patches of tall grass or weeds.) Defining the pool of potential extrapair mating partners on the basis of all nestbox residents does, however, mean that our results for any one activity state should be viewed in relation to the results for other activity states.

\section{Focal Female Dataset}

Our sample of focal females consisted of the 21 extrapair mating partners ( 24 extrapair mating events) of the focal males. For this dataset, we applied the same classification of male activity states (see above) to all males that had bred on the study site during 2000-2001 plus 2003 (range $=31-40$ males per year). We then calculated the numbers of different males (other than the female's social mate) that were in each of the four states during the fertile period of each focal female, with any males that were in an unknown state at the time excluded. (Although many of these females had more than one nesting cycle per season, we considered only fertile periods in which EPF had occurred.) We allowed individual males to occur in two different states during the fertile period of a focal female if, for example, they had been in one state at the beginning of her fertile period, and then passed into a different state while she was still fertile. We then scored the number of the female's extrapair mating partners that were in each activity state (range $=0-2$ ).

\section{Male Incubation Behaviour}

To examine the effect of variation in fertile female abundance on the incubation contributions of individual males, we used data from a previous study of biparental incubation (Bartlett et al., 2005) that had been conducted at the study site during 2000-2001. A total of 47 different pairs had been observed for that research, but following the lead of Magrath \& Elgar (1997), we excluded males that had been monitored across days in which the numbers of fertile females varied very little. This reduced the sample to 27 pairs that had each been observed on dates that differed by at least two in the number of fertile females in the study area ( $\bar{x}$ difference $=3.7 \pm 2.09$ SD females, $n=27$ ). Each pair's activities at the nestbox were monitored for one hour per morning on 2-5 days $(\bar{x}=3.1 \pm 0.53$ SD days, $n=27)$, with the number of fertile females in the 
study area averaging $5.8 \pm 2.94 \mathrm{SD}$ ( $n=85$ sample hours). We measured the incubation contributions of each pair member during each hour as the number of minutes spent inside the nestbox, and we included ambient temperature at the beginning of each hourly sample as a covariate in the analyses.

\section{Statistical Analyses}

We used SAS v. 9.2 and 9.3 (SAS Institute, Cary, NC, U.S.A.) for analyses. We relied on repeated measures linear mixed models (LMM; Proc Mixed) for most analyses of continuously distributed response variables, and repeated measures generalized linear mixed models (GLMM; Proc Glimmix) for count data (i.e., number of extrapair mates). Both Mixed and Glimmix offer several options for specifying between-subject variance as well as the nature of the correlations among multiple measurements collected on the same individual. For each model we tried several covariance structures that we considered most likely, and then used AIC values to decide which structure was most appropriate. In most cases, the default structure (variance components) yielded the best fit when coupled with individual identity as the subject; the exceptions occurred in models of the within-male changes across states in numbers of extrapair mates obtained, where we used an autoregressive structure, and in models of changes across days in the incubation behaviour of males (and females), where compound symmetry provided the best fit. We applied the Kenward-Roger method for calculations of denominator degrees of freedom for both LMMs and GLMMs. We ran analyses of the incidence of extrapair matings in relation to male activity state using both a binomial distribution (with number of matings equal to the event, and number of fertile females or number of males in that state equal to the number of trials) and a poisson distribution (with the log of the number of potential extrapair mates as an offset variable). The two approaches yielded very similar results, but models based on the poisson distribution provided a slightly better fit. The results from these analyses are presented in terms of 'incidence', meaning the mean male (or female) probability of extrapair mating per potential extrapair mate. This measure incorporates: a) the probability that the individual will encounter the potential mate; $b$ ) the probability that copulation will occur upon encounter; and c) the probability that fertilization will result 
273 from copulation. We assessed the effect of median date of each state within each cycle in models of the

274 incidence of male extrapair mating because the frequency of EPFs varies with time in season in some house

275 sparrow populations (Stewart, Hanschu, Burke \& Westneat, 2006), and because male T declines toward the

276 end of each breeding season (Hegner \& Wingfield, 1986). Least square means (LSM) have been back-

277 transformed to a linear scale. Residual analyses of the relationship between male incubation contributions

278 and numbers of fertile females indicated nonlinearity, which we corrected by log transformation. All statistical

279 tests are two-tailed.

280 Ethical Note

281

We took multiple precautions to reduce the risk that our capturing, banding, or collecting blood

282 samples would create harmful effects. For handling of adults, our procedures included continuous monitoring

283 of mistnets and trapping devices so that individuals could be removed as soon as possible after they had been

284 captured. Further, because adult females are prone to desertion if captured in their nests during laying or

285 incubation (Seel, 1968), we either captured female parents with mistnests or ground traps or we waited until

286 chicks were at least 5 days old to trap them in their nests. We nearly always used a team of two people to

287 expedite the banding and collection of blood samples from nestlings, and in cases where an entire brood

288 appeared malnourished and was undersized $(<\sim 18 \mathrm{~g})$ at normal banding age, we weighed the chicks, but

289 then returned 2-3 days later to apply bands and collect blood samples. When only one or two chicks in a

290 brood were undersized (and a later visit would potentially cause premature fledging of the remaining brood

291 members), we applied only an aluminium band for permanent identification and we reduced the size of the

292 blood sample we collected. Finally, when broods were large (4-6), we minimized disruption of parental

293 feeding by removing from the nest only half of the brood at a time for banding; when broods were small, the

294 entire brood was removed from the nest simultaneously and we left a ladder below the nestbox to deter

295 parents from returning to the nest and finding it empty. No parental abandonment of broods occurred as a

296 consequence of our handling of nestlings. Birds were captured and banded under permits issued by the 
United States Geological Survey Bird Banding Lab (Permit No. 09962) and the State of Oklahoma. This research was approved by the University of Oklahoma's Animal Care and Use Committee, Assurance Number 73-R-100.

RESULTS

Focal male schedules, realized opportunity costs, and variation in fertile female abundance

Extrapair mating events were relatively more common during the fertile and incubation stages than during the prefertile and nestling feeding stages (Fig. 2). To determine whether the occurrence of extrapair mating events differed significantly across male activity states and to estimate the relative opportunity costs of each state, we compared the number of extrapair mates males obtained during the four activity states using individually mean-centered extrapair mating success scores. Thus, if a particular male had acquired two extrapair mates in a given year, he would be expected by chance to average 0.50 mating events during each of the four states, and this analysis examined mean deviations from those averages. We found that male extrapair mating success varied significantly across states (LMM: $\left.F_{3.60 .2}=4.33, P=0.0079\right)$. Mating events occurred nonsignificantly less often than expected by chance when males were in the prefertile state or were feeding nestlings (LSM $=-0.190 \pm 0.105 \mathrm{SE} ; t_{68.3}=1.82, P=0.074$ in both cases). Their prevalence when the social mates of males were fertile did not differ significantly from that expected by chance (LSM $=0.143 \pm$ $\left.0.105 \mathrm{SE} ; t_{68.3}=1.82, P=0.178\right)$. By contrast, the numbers of extrapair matings occurring during the incubation stage of male nesting cycles were significantly higher than expected ( $\mathrm{LSM}=0.238 \pm 0.105 \mathrm{SE} ; t_{68.3}=$ $2.27, P=0.026)$.

But extrapair sires did not spend equal numbers of days in each state during the breeding season (LMM: $\left.F_{3.80}=7.76, P<0.0001\right)$, nor were the mean cumulative numbers of potential extrapair mates in each state equivalent $\left(\mathrm{LMM}: F_{3.80}=8.91, P<0.0001\right)$. Males spent more days in a prefertile state during the breeding season, and were also potentially exposed to more fertile females while in the prefertile state, than 
321 during activity states when they were involved in parental care and/or had a fertile social mate (Fig. 2). We

322 consolidated measures of the duration of each state and fertile female abundance to derive a hypothetical

323 maximum extrapair mating rate per day (the number of fertile females present during a state, divided by the

324 number of days spent in that state). This was at its highest during intervals when extrapair sires had fertile

325 social mates ( $L S M=1.4 \pm 0.07 \mathrm{SE}$ females/day), and at its lowest when males were feeding nestlings (LSM $=$

$3260.9 \pm 0.07 \mathrm{SE}$ females/day). On average, this measure of potential extrapair mating rate was significantly

327 higher when extrapair sires had fertile social mates than when they were assisting with incubation $\left(t_{80}=3.71\right.$,

$328 P=0.0004)$ or the feeding of nestlings $\left(t_{80}=4.52, P<0.0001\right)$.

Variation across states in mean extrapair matings relative to female availability

The incidence of extrapair mate acquisition (mean male extrapair matings per fertile female) changed

331 significantly across the four activity states (GLMM: $F_{3.207}=3.47, P=0.0172$ ), being conspicuously low during

332 the prefertile and feeding states, and at its highest during incubation and while a male's social mate was

333 fertile (Fig. 3). We detected no effect of the median date of male state on this pattern (GLMM: $F_{1,206}=1.26, P$

$334=0.2637)$, nor did we find any significant interaction between median date and male state (GLMM: $F_{3.203}=$

$3350.42, P=0.7407$ ). Male incidence of extrapair mating while in a prefertile state (when males are not

336 participating much in either parental care or mate-guarding) was significantly reduced relative to when males

337 were assisting with incubation $\left(t_{207}=2.80, P=0.0055\right)$ or had a fertile social mate $\left(t_{207}=2.22, P=0.0272\right)$; the

338 incidence of extrapair mating during incubation was higher than when males were feeding nestlings $\left(t_{207}=\right.$

$3392.00, P=0.0473)$.

340 Variation in numbers of males in each state when extrapair females were fertile

$341 \quad$ Focal females had similar numbers of potential extrapair partners in each state during the fertile

342 period when they participated in $\operatorname{EPF}\left(\operatorname{LMM}: F_{3,75.8}=1.59, P=0.1980\right)$; the maximum difference in the average

343 number of males in each state occurred between males that were feeding nestlings $(10.3 \pm 5.25$ SD males, $n=$

34423 fertile periods) and males that were prefertile $(12.3 \pm 4.28$ SD males, $n=23$ fertile periods). Despite this, 
the incidence of female extrapair mating varied with male state (GLMM: $F_{3,70.47}=3.82, P=0.0135$ ). In parallel

346 with the results from the focal male analysis, the females' highest incidences of extrapair mating occurred

347 with males that were participating in incubation and males with a fertile social mate, whereas their lowest

348 were with males in the prefertile state and males feeding nestlings (Fig. 3). The females' average incidence of

349 extrapair mating with prefertile males was significantly lower than their incidence of mating with males that

350 had fertile social mates $\left(t_{81.31}=2.35, P=0.0213\right)$ and males that were assisting with incubation $\left(t_{72.86}=2.63, P\right.$

$351=0.0103)$, but did not differ significantly from the incidence of mating with males feeding nestlings $\left(t_{83.26}=\right.$

$3520.23, P=0.8218)$.

353

354

355

356

357 states. The majority of extrapair matings occurred during the incubation stage and, as Wetton et al. (1995)

Male incubation contributions as predicted by fertile female abundance

Males reduced the amount of time they spent in the nestbox on mornings when fertile females were more abundant (Table 1); they also decreased the time they spent in the nest on warmer mornings. Their social mates showed a similar decline in minutes spent incubating with increased temperature, but no significant change in behaviour when fertile females were more prevalent (Table 1). As a second, very simple, assessment of male responsiveness to changes in fertile female abundance, we compared the behaviour of individual males on the day that the number of fertile females was at its minimum (of all mornings the male had been sampled) with their behaviour when the number of fertile females was at its highest. About threefourths of the males (20/26) spent fewer minutes in the nestbox on the morning when more females were fertile (Sign test: $P=0.0094$; one male with no difference in behaviour on the two days was excluded from analysis).

\section{DISCUSSION}

The extrapair mating success of focal male house sparrows changed significantly across their activity had found for this species, when an extrapair sire's social mate was fertile (see also Stewart et al. , 2006). In 
contrast, males seldom obtained extrapair matings during the prefertile and nestling feeding states. The relative rarity of matings during the prefertile state is unlikely to be attributable to a shortage of opportunities: the maximum possible extrapair mating rate for males (total number fertile females/total

372 number days) was quite high during the prefertile stage. On the other hand, fertile females were notably

373 scarce when males had nestlings to feed; even if males were to allocate equal effort during each state to extrapair mate acquisition, we would expect them to accumulate fewer matings during nestling feeding.

375 Similarly, fertile females also were less abundant during incubation than when the males' social mates were 376 fertile. Thus, as Knowlton (1979) concluded, breeding synchrony reduces the opportunity costs of male 377 parental care by decreasing the number of matings potentially available to parenting males; males simply are not missing out on as much during intervals when they are providing care.

These analyses based on sheer numbers of events occurring reflect variation across stages of the cycle in realized opportunity costs, but they are not particularly informative about which male activities may impose constraints on male mating success because they do not take into account the supply of potential mating opportunities. Comparisons of the mean male incidence of extrapair mating revealed that the extent to which males capitalize on mating opportunities changes substantially across states. When males have a fertile social mate or are assisting with incubation, they seem to be better at converting potential extrapair mating partners into actual matings than when they are in a prefertile state or have nestlings to feed.

386 Moreover, these state-related changes in the incidence of extrapair mating do not appear to originate from a 387 biased pool of prospective extrapair partners during the fertile periods of females that engage in extrapair matings. Such biases could arise if female participants in EPF breed at times of especially high synchrony, such 389 that most of their potential mating partners would be males with fertile social mates, or shortly after high 390 synchrony periods, when incubating males would be particularly abundant. This was not the case, however, and consequently, we conclude that either females that engage in extrapair matings prefer males in particular 
states, and/or males in certain states are expending more effort, or more productive effort, in obtaining extrapair fertilizations.

These results point to two activity states of male house sparrows that feature unusually low probabilities of mating per potential opportunity, such that males can be considered to be in partial time-out

396 from the extrapair mating pool. This partial time-out during the prefertile state creates high opportunity costs 397 for males, because fertile females are plentiful. We can think of no particularly cogent reason why males that 398 are known to be capable of extrapair mating are so ineffective at it during inter-brood intervals or when they 399 lack an actively-reproducing social mate. For example, compared to periods when they have a fertile social 400 mate and are subject to sperm depletion (Birkhead, Veiga, \& Møller, 1994), one might expect any extrapair copulations they engage in to have a high chance of fertilization. Furthermore, except toward the end of a 402 breeding season, male T levels are relatively high during interbrood intervals or when males are competing 403 for social mates and nesting sites (Hegner \& Wingfield, 1986; Schwagmeyer et al., 2005). Thus, to the extent 404 that T supports phenotypic traits that facilitate encountering, copulating with, and fertilizing prospective 405 extrapair partners, the low success of these males is paradoxical. Perhaps there is a greater conflict between 406 extrapair mating effort and effort directed toward acquiring or retaining a social mate plus nesting site than is 407 generally acknowledged. Alternatively, males may routinely face greater competition for extrapair matings 408 when they are in a prefertile state. Our ability to evaluate this hypothesis is limited. Although the focal female 409 results indicate that the numbers of males at the study site that were in each state were roughly equivalent 410 when extrapair matings occurred, we do not know how many males in each state were actually attempting to 411 acquire extrapair matings. In any case, the low success of prefertile males appears not to be unique to our 412 study site: Wetton et al. (1995) also commented on how rarely extrapair matings occurred when house 413 sparrow males were not undergoing a nesting cycle. A reduced likelihood of capitalizing on extrapair mating opportunities when males have nestlings to

415 feed is more easily understood, and has additionally been shown to exist in pied flycatchers, Ficedula 
hypoleuca (Canal, Jovani, \& Potti, 2012). The depression of male efficiency at extrapair mating at this time is

417 consistent with traditional views regarding a trade-off between parental effort and mating effort, as well as

418 the hypothesis that this trade-off is mediated by T (Ketterson and Nolan, 1994; Wingfield, Hegner, Dufty, \&

419 Ball, 1990). Male and female house sparrow parents provision their young at very similar rates for about the

420 first 10 days of the nestling stage (Hegner \& Wingfield, 1987a; Mock, Schwagmeyer, \& Parker, 2005), and

421 male T levels are low during this interval (Hegner \& Wingfield, 1986; 1987a; Schwagmeyer et al., 2005).

422 Moreover, testosterone-induced reductions in male provisioning levels confirm that male contributions

423 substantially enhance the welfare of their offspring (Hegner \& Wingfield, 1987b; Mazuc, Chastel, \& Sorci,

424 2003; Schwagmeyer et al., 2012). Thus, male care during the nestling stage appears to be both time-

425 consuming and highly valuable in this species.

426

427

the incubation stage (Bartlett et al., 2005). The discrepancies in male time commitments to incubation versus

428

nestling feeding consequently parallel the differences between these states in opportunity costs and the

relative proficiency of males in acquiring EPFs. Furthermore, while male house sparrows probably play a

crucial role in defending the nest from competitors during the incubation stage (Schiefferli, 1978), male

incubation per se may not be as valuable as male provisioning: males are able to maintain nest temperatures

during their incubation bouts, but they are not as capable as females at increasing nest temperatures (Parker et al., 2014). This sexual asymmetry in incubation effectiveness is sufficient to promote the evolution of sex role divergence in time commitments, with sex differences in opportunity costs as a non-mutually exclusive 435 alternative (Parker et al., 2014). reason for the high incidence of male extrapair mating during this state. We tested whether males might also be enhancing their success at this stage by modifying their incubation contributions in response to daily 
the amount of time they spend in the nest on mornings when more females are fertile, as Magrath \& Elgar

441 (1997) had found for fairy martins, Hirundo ariel. Females of neither species, by contrast, showed any

442 significant change in their contributions, although female house sparrows compensate partially for

443 testosterone-induced reductions in male incubation (Schwagmeyer et al., 2005), and unmanipulated females

444 typically extend their bout durations slightly when their mate takes a lengthy recess (Schwagmeyer et al., 445 2008).

At least two other approaches have been used to detect and define the nature of the opportunity

447 costs that are associated with avian male reproductive activities. Thomson et al. (2014) quantified the opportunity costs of male incubation by using naturally occurring variation in the parental behaviour of

449 Temminck's stints, Calidris temminckii, a species in which either sex provides uniparental care. They found 450 that males that provide no parental care during a season sire more offspring from EPFs, stored sperm, or brief 451 pairings with care-giving females than males that undertake care of a clutch. In a second approach, male 452 parental care has been manipulated via testosterone implants to assess whether full participation in care 453 diminishes male success in acquiring alternative matings. In dark-eyed juncos, Junco hyemalis, T-implanted 454 males achieve more EPFs than control males (Raouf, Parker, Ketterson, Nolan, \& Ziegenfus, 1997; Reed et al., 455 2006), and in white-crowned sparrows, Zonotrichia leucophrys pugentensis, and song sparrows, Melospiza 456 melodia, T-treatment increases the incidence of polygynous pairings (Wingfield, 1984). Testosterone manipulations of male house sparrows also revealed reduced chances of acquiring 458 additional social mates as an opportunity cost of male parental care (Schwagmeyer et al., 2012). However, we 459 detected no significant effect of T-treatment on the EPF success of male house sparrows, leading us to 460 suggest that perhaps most extrapair mating opportunities occur when males are in a high-T state anyway, and 461 providing little or no parental care. The results on variation in fertile female abundance from this study 462 support that hypothesis: focal males had more extrapair mating prospects when they were in the prefertile 463 and fertile states than when they were parenting. But in addition, the results show that, despite their having 
high T levels, unmanipulated males seldom acquire extrapair mates during intervals when they are engaged in 465 attracting or retaining a social mate and a nesting site (i.e., the prefertile state). Our experimental T males 466 that were attempting to attract secondary social mates may consequently have been restricting their 467 extrapair mating success. Regardless, by focusing here on the subset of 'elite' males that are known to engage 468 in extrapair mating, we have found that male care in the form of nestling provisioning appears to detract from 469 male effectiveness in acquiring extrapair mates, whereas male contributions to incubation seem not to.

471 Acknowledgments

472 Parentage analyses were conducted in the laboratory of Dr. Patricia G. Parker at the University of Missouri at 473 St. Louis. We thank Patty for her supervision of that work and Charlotte Roy, Noah Whiteman, Cintia 474 Cornelius, John Eimes, Shane Siers, Jennifer Bolmer, Karen DeMatteo, Sarah Shrock and Cindee Rettke for 475 their assistance with the analyses. We are grateful to Jennifer Alig, Christine Edly-Wright, Amy Kopisch, Chris 476 Leary, Bonnie Means, Austen Patterson, Lace Svec, Taryn West and Lindsay White for their contributions to 477 the North Base field work. We also thank three anonymous reviewers for their comments on the manuscript, 478 and Ken Yasukawa for his editorial assistance. This research was supported by the National Science 479 Foundation (IBN-9982661), a George M. Sutton Scholarship in Ornithology, and a Blanche Adams Scholarship.

481 References

482

483 Akçay, Ç., Searcy, W. A., Campbell, S. E., Reed, V. A., Templeton, C. N., Hardwick, K. M., \& Beecher, M. D. 484 (2011). Who initiates extrapair mating in song sparrows? Behavioral Ecology. doi: 10.1093/beheco/arr147 485 Bartlett, T. L., Mock, D. W., \& Schwagmeyer, P. L. (2005). Division of labor: Incubation and biparental care in 486 house sparrows (Passer domesticus). Auk, 122(3), 835-842. 
Birkhead, T. R., Veiga, J. P., \& Møller, A. P. (1994). Male sperm reserves and copulation behaviour in the house sparrow, Passer domesticus. Proceedings of the Royal Society of London B: Biological Sciences, 256(1347), 247-251.

Canal, D., Jovani, R., \& Potti, J. (2012). Male decisions or female accessibility? Spatiotemporal patterns of extra pair paternity in a songbird. Behavioral Ecology, 23(5), 1146-1153. doi: 10.1093/beheco/ars090

Clutton-Brock, T. H., \& Parker, G. A. (1992). Potential reproductive rates and the operation of sexual selection. The Quarterly Review of Biology, 67(4), 437-456.

Edly-Wright, C., Schwagmeyer, P. L., Parker, P. G., \& Mock, D. W. (2007). Genetic similarity of mates, offspring health and extrapair fertilization in house sparrows. Animal Behaviour, 73(2), 367-378.

Forstmeier, W. (2007). Do individual females differ intrinsically in their propensity to engage in extra-pair copulations? PLoS ONE, 2(9), e952.

Grafen, A. (1980). Opportunity cost, benefit and degree of relatedness. Animal Behaviour, 28(AUG), 967-968. doi: $10.1016 / \mathrm{s} 0003-3472(80) 80160-6$

Hammers, M., von Engelhardt, N., Langmore, N. E., Komdeur, J., Griffith, S. C., \& Magrath, M. J. L. (2009). Mate-guarding intensity increases with breeding synchrony in the colonial fairy martin, Petrochelidon ariel. Animal Behaviour, 78(3), 661-669. doi: http://dx.doi.org/10.1016/j.anbehav.2009.06.013

Hegner, R. E., \& Wingfield, J. C. (1986). Behavioral and endocrine correlates of multiple brooding in the semicolonial house sparrow Passer domesticus I. Males. Hormones and Behavior, 20(3), 294-312. doi: http://dx.doi.org/10.1016/0018-506X(86)90039-5

Hegner, R. E., \& Wingfield, J. C. (1987a). Effects of brood-size manipulations on parental investment, breeding success, and reproductive endocrinology of house sparrows. Auk, 104(3), 470-480. doi: 10.2307/4087546

Hegner, R. E., \& Wingfield, J. C. (1987b). Effects of experimental manipulation of testosterone levels on parental investment and breeding success in male house sparrows. Auk, 104(3), 462-469. 
Ketterson, E. D., \& Nolan, V. (1994). Male parental behavior in birds. Annual Review of Ecology and Systematics, 25, 601-628. doi: 10.1146/annurev.es.25.110194.003125

Knowlton, N. (1979). Reproductive synchrony, parental investment, and the evolutionary dynamics of sexual selection. Animal Behaviour, 27, Part 4(0), 1022-1033. doi: http://dx.doi.org/10.1016/00033472(79)90049-6

Kokko, H., \& Jennions, M. D. (2008). Parental investment, sexual selection and sex ratios. Journal of Evolutionary Biology, 21(4), 919-948. doi: 10.1111/j.1420-9101.2008.01540.x

Kokko, H., Klug, H., \& Jennions, M. D. (2012). Unifying cornerstones of sexual selection: operational sex ratio, Bateman gradient and the scope for competitive investment. Ecology Letters, 15(11), 1340-1351. doi: 10.1111/j.1461-0248.2012.01859.x

Magrath, M. J. L., \& Elgar, M. A. (1997). Paternal care declines with increased opportunity for extra-pair matings in fairy martins. Proceedings of the Royal Society of London Series B-Biological Sciences, 264(1389), 1731-1736.

Magrath, M. J. L., \& Komdeur, J. (2003). Is male care compromised by additional mating opportunity? Trends in Ecology \& Evolution, 18(8), 424-430. doi: http://dx.doi.org/10.1016/S0169-5347(03)00124-1

Mazuc, J., Chastel, O., \& Sorci, G. (2003). No evidence for differential maternal allocation to offspring in the house sparrow (Passer domesticus). Behavioral Ecology, 14(3), 340-346. doi: 10.1093/beheco/14.3.340

Mock, D. W., Schwagmeyer, P. L., \& Parker, G. A. (2005). Male house sparrows deliver more food to experimentally subsidized offspring. Animal Behaviour, 70, 225-236.

Neudorf, D. L., Stutchbury, B. J. M., \& Piper, W. H. (1997). Covert extraterritorial behavior of female hooded warblers. Behavioral Ecology, 8(6), 595-600. doi: 10.1093/beheco/8.6.595

Parker, G. A., Schwagmeyer, P. L., \& Mock, D. W. (2014). The asymmetric incubation game: a prospective model and a house sparrow investigation. Animal Behaviour, 93(0), 37-47. doi:

http://dx.doi.org/10.1016/j.anbehav.2014.04.012 
Parker, G. A., \& Simmons, L. W. (1996). Parental investment and the control of sexual selection: Predicting the direction of sexual competition. Proceedings of the Royal Society of London Series B-Biological Sciences, 263(1368), 315-321. doi: 10.1098/rspb.1996.0048

Raouf, S. A., Parker, P. G., Ketterson, E. D., Nolan, V., \& Ziegenfus, C. (1997). Testosterone affects reproductive success by influencing extra-pair fertilizations in male dark-eyed juncos (Aves: Junco hyemalis). Proceedings of the Royal Society of London Series B-Biological Sciences, 264(1388), 1599-1603.

Reed, W. L., Clark, M. E., Parker, P. G., Raouf, S. A., Arguedas, N., Monk, D. S., Snajdr, E., Nolan, V., \& Ketterson, E. D. (2006). Physiological effects on demography: A long-term experimental study of testosterone's effects on fitness. American Naturalist, 167(5), 667-683. doi: 10.1086/503054

Schiefferli, L. (1978). Die rolle des männchens während der bebrütung der eier beim hausperling Passer domesticus. Ornithologische Beobachter, 75(1), 44-47.

Schwagmeyer, P. L. (2014). Partner switching can favour cooperation in a biological market. Journal of Evolutionary Biology, 27(9), 1765-1774. doi: 10.1111/jeb.12455

Schwagmeyer, P. L., Bartlett, T. L., \& Schwabl, H. G. (2008). Dynamics of house sparrow biparental care: what contexts trigger partial compensation? Ethology, 114(5), 459-468. doi: 10.1111/j.1439-0310.2008.01480.x

Schwagmeyer, P. L., Parker, P. G., Mock, D. W., \& Schwabl, H. (2012). Alternative matings and the opportunity costs of paternal care in house sparrows. Behavioral Ecology, 23(5), 1108-1114. doi:

10.1093/beheco/ars080

Schwagmeyer, P. L., Schwabl, H. G., \& Mock, D. W. (2005). Dynamics of biparental care in house sparrows: hormonal manipulations of paternal contributions. Animal Behaviour, 69, 481-488. doi: 10.1016/j.anbehav.2004.04.017

Seel, D. C. (1968). Clutch size, incubation and hatching success in the house sparrows and tree sparrow spp. at Oxford. Ibis, 110(3), 270-282. doi: 10.1111/j.1474-919X.1968.tb00038.x 
Simmons, L. W., \& Parker, G. A. (1996). Parental investment and the control of sexual selection: Can sperm competition affect the direction of sexual competition? Proceedings of the Royal Society of London. Series B: Biological Sciences, 263(1370), 515-519. doi: 10.1098/rspb.1996.0078

Stewart, I. R. K., Hanschu, R. D., Burke, T., \& Westneat, D. F. (2006). Tests of ecological, phenotypic, and genetic correlates of extra-pair paternity in the house sparrow. The Condor, 108(2), 399-413. doi: 10.1650/0010-5422(2006)108[399:toepag]2.0.co;2

Stiver, K. A., \& Alonzo, S. H. (2009). Parental and mating effort: Is there necessarily a trade-off? Ethology, 115(12), 1101-1126. doi: 10.1111/j.1439-0310.2009.01707.x

Stutchbury, B. J. M. (1998). Female mate choice of extra-pair males: breeding synchrony is important. Behavioral Ecology and Sociobiology, 43(3), 213-215. doi: 10.1007/s002650050483

Tallamy, D. W. (2000). Sexual selection and the evolution of exclusive paternal care in arthropods. Animal Behaviour, 60(5), 559-567. doi: http://dx.doi.org/10.1006/anbe.2000.1507

Thomson, R. L., Pakanen, V. M., Tracy, D. M., Kvist, L., Lank, D. B., Ronka, A., \& Koivula, K. (2014). Providing parental care entails variable mating opportunity costs for male Temminck's stints. Behavioral Ecology and Sociobiology, 68(8), 1261-1272. doi: 10.1007/s00265-014-1737-4

Veiga, J. P. (1990). Sexual conflict in the house sparrow: interference between polygynously mated females versus asymmetric male investment. Behavioral Ecology and Sociobiology, 27(5), 345-350. doi: $10.1007 /$ bf00164005

Weatherhead, P. J. (1997). Breeding synchrony and extra-pair mating in red-winged blackbirds. Behavioral Ecology and Sociobiology, 40(3), 151-158. doi: 10.1007/s002650050328

Westneat, D. F., Sherman, P. W., \& Morton, M. L. (1990). The ecology and evolution of extra-pair copulations in birds. Current ornithology, 7, 331-369.

Westneat, D. F., \& Gray, E. M. (1998). Breeding synchrony and extrapair fertilizations in two populations of red-winged blackbirds. Behavioral Ecology, 9(5), 456-464. doi: 10.1093/beheco/9.5.456 
581 Wetton, J. H., Burke, T., Parkin, D. T., \& Cairns, E. (1995). Single-locus DNA fingerprinting reveals that male 582 reproductive success increases with age through extra-pair paternity in the House Sparrow (Passer 583 domesticus). Proceedings: Biological Sciences, 260(1357), 91-98.

584 Whittingham, L. A., Dunn, P. O., \& Stapleton, M. K. (2006). Repeatability of extra-pair mating in tree swallows. 585 Molecular Ecology, 15(3), 841-849. doi: 10.1111/j.1365-294X.2006.02808.x

586 Wingfield, J. C. (1984). Androgens and mating systems - testosterone-induced polygyny in normally 587 monogamous birds. Auk, 101(4), 665-671.

588 Wingfield, J. C., Hegner, R. E., Dufty, A. M., \& Ball, G. F. (1990). The challenge hypothesis - theoretical 589 implications for patterns of testosterone secretion, mating systems, and breeding strategies. American $590 \quad$ Naturalist, 136(6), 829-846. doi: 10.1086/285134

591 
592 Table 1

593 Numbers of $\mathrm{min} / \mathrm{hr}$ spent in nestbox by parents of each sex as predicted by numbers of fertile females at the

594 study site and ambient temperature

595

596

Males

\begin{tabular}{lccccc} 
Effect & Estimate & SE & DF & $t$ & $P$ \\
\cline { 2 - 6 } Intercept & 21.55 & 3.095 & 75.2 & 6.96 & $<0.0001$ \\
log\# females & -4.63 & 1.720 & 81.9 & -2.69 & 0.0087 \\
Temperature & -0.41 & 0.196 & 79.6 & -2.10 & 0.0387
\end{tabular}

Females

\begin{tabular}{lrrrrc} 
Effect & Estimate & SE & DF & $t$ & $P$ \\
\cline { 2 - 6 } Intercept & 27.57 & 3.282 & 72.5 & 8.40 & $<0.0001$ \\
log\# females & 2.01 & 1.826 & 75.5 & 1.10 & 0.2742 \\
Temperature & -0.48 & 0.206 & 79.4 & -2.33 & 0.0225
\end{tabular}

599 Temperature was mean-centred at the average temperature at the onset of the 85 sample hours $\left(19.96^{\circ} \mathrm{C}\right)$. 


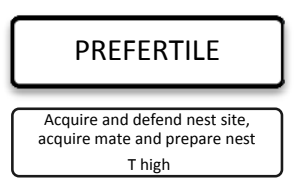
Beginning of breeding season up to
onset of mate's fertile period

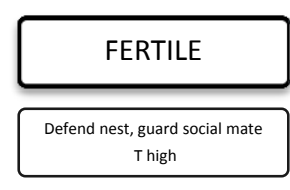

5 days before first egg laid through

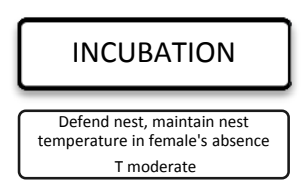

Day after clutch completion through

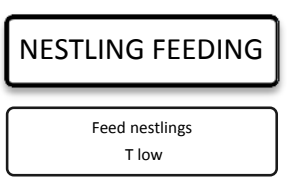

Day after hatching through next 10

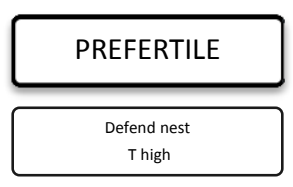

Day 12 of nestling period until next
female fertile period or breeding female fertile period or breeding
600

601

602

603

604

605

606

607
Figure 1. Sequence of male activity states for a case where a male begins the breeding season without a social mate, undergoes one nesting cycle, and then enters another prefertile state before his next nesting cycle. The second row of boxes provide descriptive information about male activities at the nest site (based on Hegner \& Wingfield, 1986 or references cited in text) and average male T levels (based on Hegner \& Wingfield, 1986, 1987a and Schwagmeyer, Schwabl, \& Mock, 2005). Timing and duration of each activity state are given in the third row of boxes. 


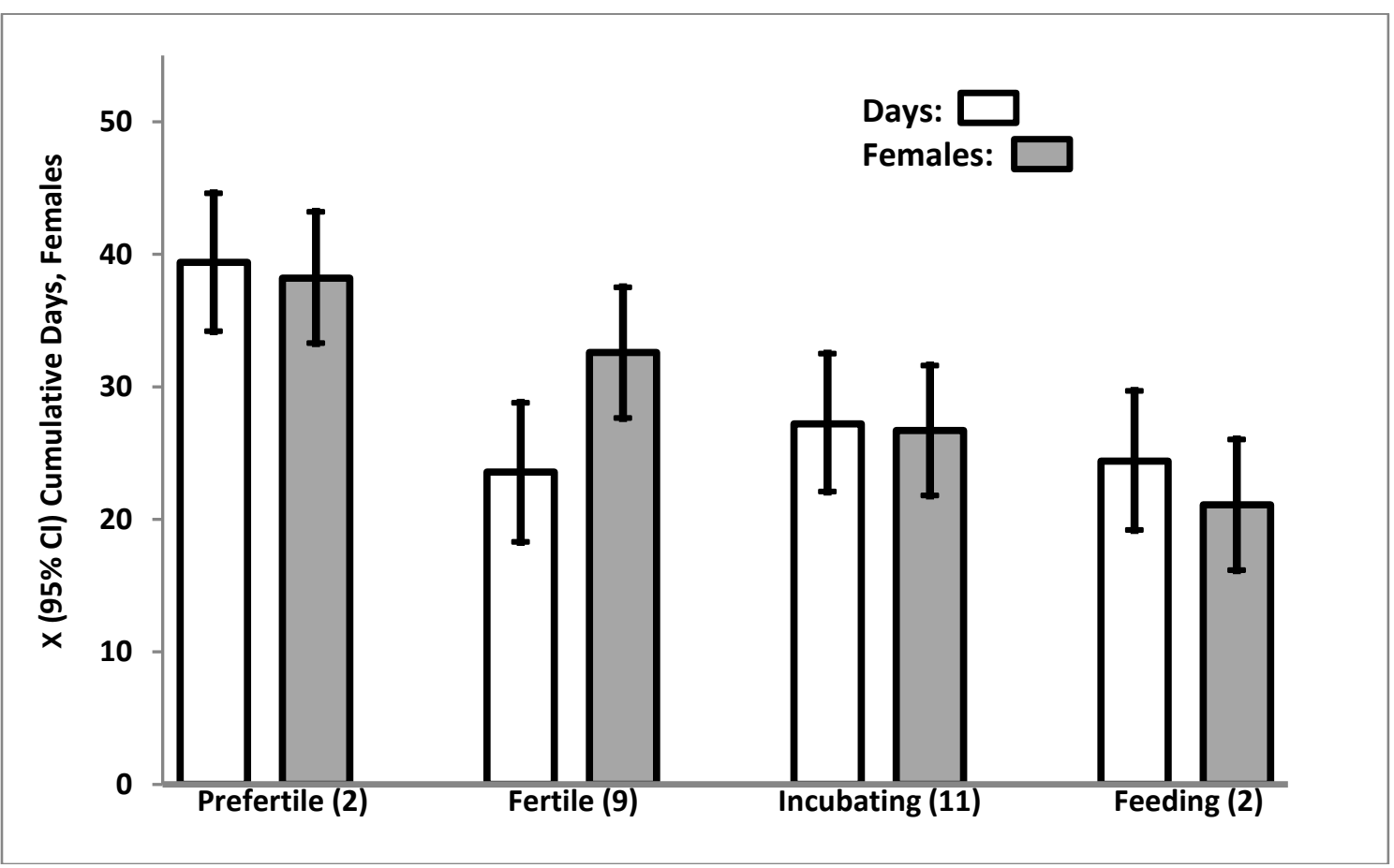

Figure 2. Mean (+95\% Cl) cumulative days (light bars) spent by focal males in prefertile, fertile, incubating, and feeding states during a breeding season, and mean (+95\% Cl) cumulative number of females (dark bars)

614 that were fertile during those states. Numbers of extrapair mates acquired in each state are indicated in 615 parentheses.

616 


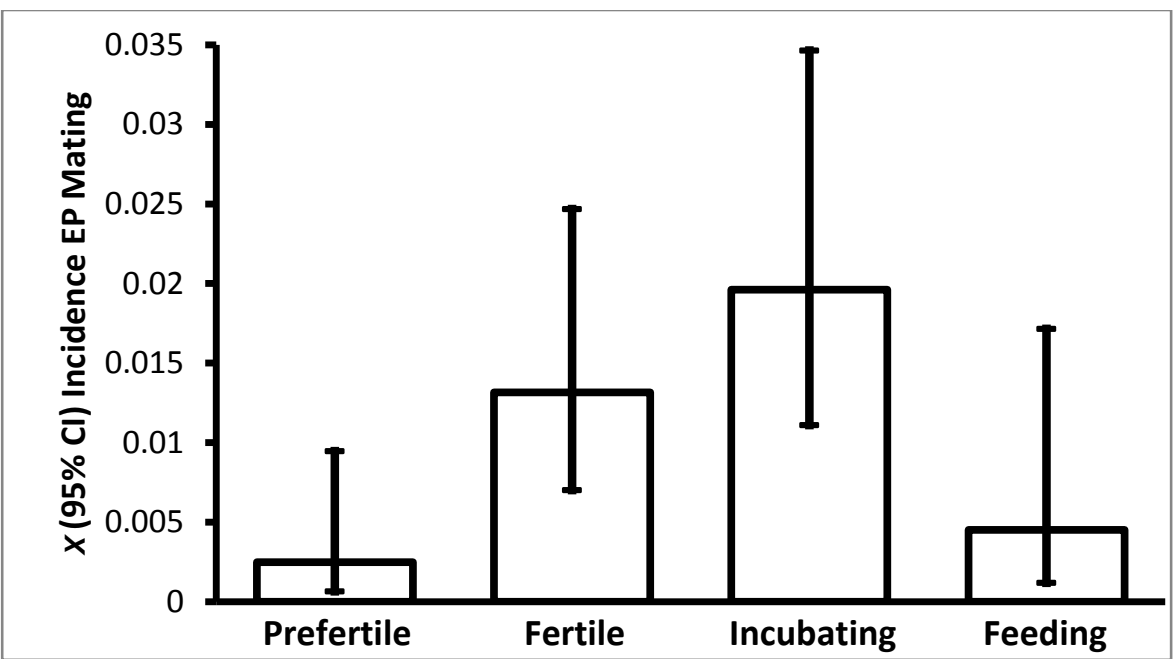

618

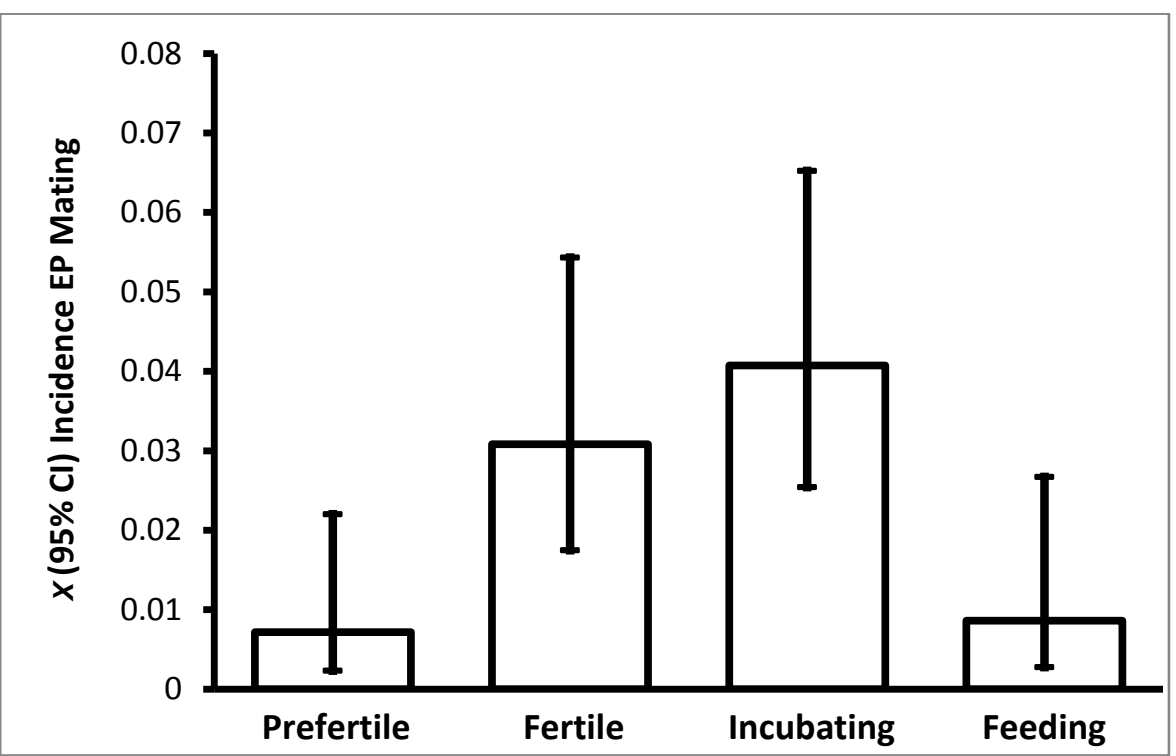

620

621

622 Figure 3. Top: Least squares mean $(+95 \% \mathrm{Cl})$ focal male incidence of extrapair mating per fertile female

623 during each activity state. Bottom: Least squares mean (+95\% $\mathrm{Cl})$ focal female incidence of extrapair mating 624 per male in each activity state during the female's fertile period.

625

626

627 